\section{FACTORS ASSOCIATED WITH QUALITY IMPROVEMENT IN LIGHT OF CHANGING PAYMENT SOURCES IN NURSING HOMES}

J. Gaudet Hefele ${ }^{1}$, J. Wang ${ }^{1}$, A. Barooah ${ }^{1}$, C.E. Bishop ${ }^{2}, 1$. UMass Boston, Melrose, Massachusetts, 2. Heller School for Social Policy and Management, Brandeis University, Waltham, Massachusetts

Attractive Medicare payments and new Medicare payment reforms create strong incentives for nursing homes (NHs) to increase their Medicare resident census, but little is known about the possible impact these increases have on quality. While higher Medicare payments might be used for quality improvement, multitasking theory suggests increased attention to one area of care (short-stay care for Medicare patients) can have negative effects on other, unrewarded areas (care for long stay residents). Using data from 20052010 (LTCFoucUS.org, Nursing Home Compare, and the Area Health Resources File; approximately 14,500 NHs in each year), we sought to determine whether increased Medicare census was associated with a decrease in quality. Our study found that on average, increased Medicare census in the previous year was associated with modest quality improvement in the next year for a number long-stay care quality indicators. However, this pattern was not universal and preliminary findings suggest it may vary systematically across different types of nursing homes, such as ownership type. Our next phase of analysis will identify which nursing home characteristics are associated with being more likely to maintain or improve quality in response to increased Medicare census, and identify other characteristics associated with being more likely to experience a decline in quality.

\section{SESSION 3875 (SYMPOSIUM)}

\section{CO-HOST ASA: NUTRITION FOR HEALTHY AGING- POLICY AND PRACTICE}

Chair: B. Blancato, American Society on Aging, San

Francisco, California

Co-Chair: M. Ponder, Matz, Blancato and Associates, Washington, District of Columbia

Food and nutrition become ever more important to humans as we age as a social and health support for all our ailments. Aging services can provide food as socialization; programs like congregate dining and home-delivered meals constitute wellness checks and stave off depression. Therapeutic nutrition through healthcare providers and increasingly through aging services programs can provide food as medicine to treat and prevent various conditions. This symposium will examine U.S. society's policies and practices around these social and medical nutrition supports. Where are the gaps in these supports? What challenges do nutrition aging services providers face, both in the field and from their for-profit competitors? Why are seniors in the United States food insecure and/or malnourished? What policies can lawmakers support in order to ensure that no older adult in the United States is hungry or malnourished? These questions and more will be discussed during this symposium.

\section{MAINTAINING SERVICE PROVISION IN AN AGE OF AUSTERITY}

P. Downey, Serving Seniors, San Diego, California

Many aging services providers in the United States today provide an array of services to the community beyond nutrition, including housing, oral health, physical and mental healthcare, legal aid, and education. However, providing this universe of services in an age of lowered federal funding and competition from for-profit healthcare and other services providers can mean that these not-for-profit aging services providers find it difficult to maintain full funding for even their core missions of nutrition and other basic services provision. This presentation will focus on this challenge and will discuss solutions from the field and from local, state and federal policy.

\section{A NEW MULTIDISCIPLINARY COALITION TO COMBAT OLDER ADULT MALNUTRITION}

M. Ponder, B. Blancato, Matz, Blancato and Associates, Washington, District of Columbia

Malnutrition is a nutrition imbalance that affects both overweight and underweight patients: in particular, older adult patients. In the acute care hospital setting, it affects approximately 20 to 60 percent of admitted patients. Nearly 35-50 percent of older residents in long term care facilities are malnourished. Chronic disease increases the risk of malnutrition in older adults. While older adult malnutrition is a prevalent and potentially costly problem, it is also preventable. Effective and timely screening is essential to help providers make accurate diagnoses, and early nutrition interventions have been shown to substantially reduce readmission rates, as well as complication rates, length of stay, cost of care, and in some cases, mortality. This presentation will describe the formation of a multidisciplinary coalition, DefeatMalnutrition.Today. The coalition seeks to increase awareness of the problem of older adult malnutrition and to propose policy solutions to combat this growing crisis. The coalition has already taken steps to change policy. This coalition is unique in its multidisciplinary nature, bringing together nutrition, wellness, faith, aging, health, business, and other groups. This is an important health policy opportunity for the gerontology network to become involved in a serious health problem.

\section{AGING AND NUTRITION: WHERE WE'VE BEEN AND WHERE WE'RE GOING}

M. Raimondi, Academy of Nutrition and Dietetics,

Washington, District of Columbia

The U.S. has made great strides in the last few years in older adult nutrition policy, in both regulations and legislation. This presentation will detail these advances and discuss what they mean for older adults. Also, with a new U.S. President in 2017 come new challenges and opportunities. This presentation will discuss the new Administration and its potential goals, as well as the new Congress and potential legislation surrounding senior nutrition, hunger and food insecurity, including the renewals of the Farm Bill and the Older Americans Act. It will also focus on the ways in which gerontologists and others in the field of aging can impact this new policy. 